Radial and Nonradial Pulsations as Probes of Stellar Physics

ASP Conference Series, Vol. 259, 2002

C. Aerts, T.R. Bedding, \& J. Christensen-Dalsgaard, eds.

\title{
Spectroscopy of Rapidly Oscillating Ap Stars
}

\author{
W.W. Weiss ${ }^{1}$, T.A. Ryabchikova ${ }^{1,2}$, I. Savanov ${ }^{3}$, N. Piskunov ${ }^{4}$, \\ V. Tsymbal ${ }^{5}$, P. Mittermayer ${ }^{1}$, P. Martinez ${ }^{6}$, O. Kochukhov ${ }^{4}$, \\ N. Nesvacil ${ }^{1}$ \\ ${ }^{1}$ Institute for Astronomy, Univ. Vienna, Türkenschanzstrasse 17, \\ A-1180Vienna, Austria (last_name@astro.univie.ac.at) \\ ${ }^{2}$ Institute of Astronomy, Russ. Acad. Sciences, Moscow, Russia \\ (ryabchik@inasan.rssi.ru) \\ ${ }^{3}$ Crimean Astrophysical Observatory, c/o 334413 Nauchny, Crimea, \\ Ukraine (savanov@crao.crimea.ua) \\ ${ }^{4}$ Uppsala Astronomical Observatory, S-751 20 Uppsala, Sweden \\ (last_name@astro.uu.se) \\ ${ }^{5}$ Tavrian National University, Physics Dept., 950000 Crimea, Ukraine \\ (vad@ccssu.crimea.ua) \\ ${ }^{6}$ South African Astronomical Observatory, P.O. Box 9, Observatory \\ 7935, South Africa (peter@saao.ac.za)
}

\begin{abstract}
We identify spectroscopic differences between roAp stars and Ap stars with no observational evidence for pulsation, but with otherwise similar $T_{\text {eff }}$ and $\log g$ values. These differences concern the abundance pattern, Hydrogen line profile anomalies, evidence for stratification, and effects of pulsation on spectral lines.
\end{abstract}

\section{Introduction}

The detection of rapid oscillation in HD 101065 by D. Kurtz in 1978 marked the discovery of a new class of pulsating stars, the roAp stars, and provided an important impetus to asteroseismology. Contrary to the other pulsating variables known at that time, roAp stars are a subgroup of already quite 'abnormal' stars, the well-known but still not well understood chemically peculiar A-type stars. Their peculiar atmosphere causes considerable problems in determining stellar fundamental parameters, like $T_{\text {eff }}, \log g$ or metallicity with classical techniques calibrated with 'normal' stars. As we had been developing the tools for a better understanding of $\mathrm{CP}$ star atmospheres by replacing various approximations by more physical descriptions, we decided to investigate roAp stars spectroscopically and to determine their fundamental parameters, in particular their abundance spectrum relative to the sun and to non-pulsating Ap (noAp) stars with similar $T_{\text {eff }}$ and $\log g$.

\section{Spectroscopic signature of roAp stars}

In $1996 \alpha$ Cir was the only roAp star with a well investigated atmosphere (Kupka et al., 1996). Thanks to the collaboration of many experts at various institutions, availability of already archived spectra and granting of telescope time, the 
situation has changed significantly, which, unfortunately, is not fully reflected in the limited size of the present list of authors.

Atmospheric characteristics of 12 out of 32 known roAp stars are now available, at least for the most important group of elements, and including such a pathological case as Przybylski's star (Cowley et al., 2000). A synopsis of the average abundance pattern found is given in Fig. 1 where we compare roAp stars with Ap stars showing no indication for pulsation.

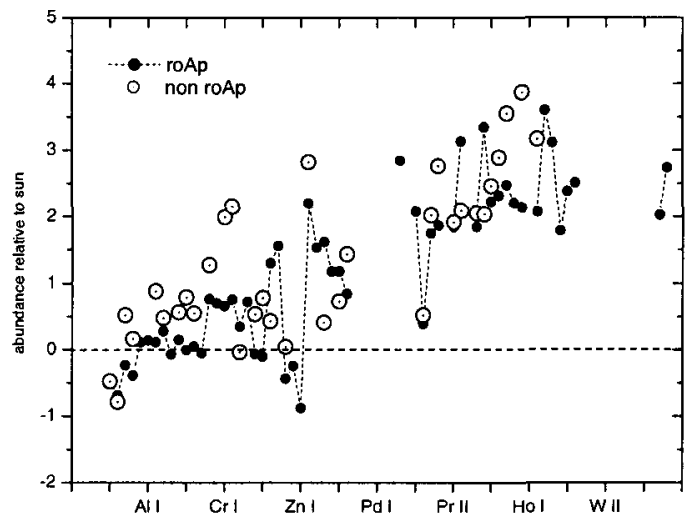

Figure 1. Average abundances for roAp stars and Ap stars with no indication for pulsation, but in the same range of $T_{\text {eff }}$ and $\log g$.

Based on these data we can characterize the spectroscopic signature of roAp stars as follows.

- Abundance pattern: All roAp stars have a $\mathrm{Cr}$ abundance lower by about 1.4 dex relative to their non-pulsating counterparts. Similar discrepancies may exist for a few other elements, but not as pronounced and with the need of confirmation. A tendency exists for hotter roAp stars to have higher iron-peak element abundances but lower rare-earth element (REE) abundances. The spectrum of the cool Przybylski's star is dominated by lines of singly and doubly ionised rare-earth lines, while the usual lines of iron-group elements are abnormally weak. We found stars similar to Przybylski's star towards the hot end of the roAp star group: HD 137949, HD 166473, and HD 12098 which have the highest REE abundances among the roAp stars.

- Hydrogen line profile anomaly: $\mathrm{H} \alpha$ and $\mathrm{H} \beta$ profiles of roAp stars show steeper cores than non-pulsating Ap and 'normal' stars and in addition some flux excess at the transition from core to wing (Cowley et al., 2001). Within the context of an LTE calculation, the authors have been unable to reproduce both the deep, narrow cores and the wings and several numerical experiments with blanketed 


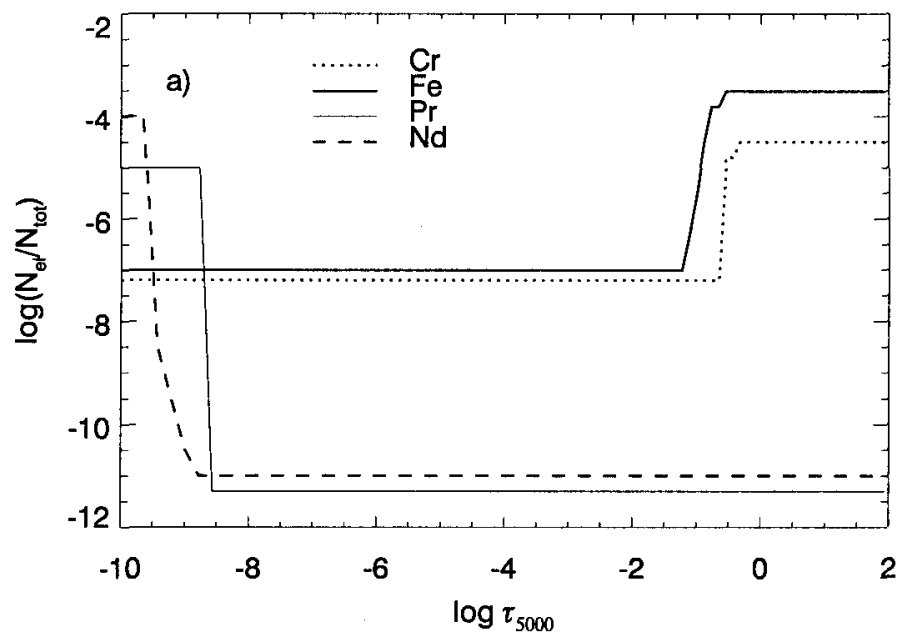

Figure 2. Abundance stratification of some chemical elements through the atmosphere of $\gamma$ Equ.

models were investigated. Even with reduced boundary temperatures it was impossible to reproduce the deep $\mathrm{H} \alpha$ core without at the same time causing nearby low-excitation metal lines become unrealistically strong well before the $\mathrm{H} \alpha$ core becomes comparably deep to the observations. It remains to be seen if these profiles can be reproduced by arbitrary modifications of the temperature distribution that might be attributed to convection, or convection in a magnetic, pulsating atmosphere.

- Evidence for stratification: Evidence for abundance stratification (see Fig. 2) in the atmospheres of chemically peculiar stars are:

- core and wings of strong saturated lines are difficult to fit with a single abundance value,

- the microturbulence velocity parameter obtained in a standard abundance analysis is different for various elements and it can be even negative for some elements in non-magnetic peculiar stars (e.g. in Hg-Mn stars),

- abundances obtained from weak lines deviate oppositely to what would be expected from an incorrectly chosen microturbulence from strong lines of the same ion,

-abundances obtained from lines of different ionization stages are discordant. This effect is particularly significant for the REE Pr and Nd where abundances determined from lines of the third spectrum appear to be overabundant by about 1 dex (!!) relative to singly ionized stages. The suspicion that this effect might be caused by incorrect atomic data is contradicted by the fact that a similar analysis of non-pulsating Ap stars with otherwise similar properties do not show this imbalance.

Individual discrepant abundances determined from various lines can be, of course, removed by modifying atmospheric parameters, but in some cases such 
a model does not even fit the observed energy distribution, photometric indices or hydrogen line profiles. One simply fails to find a unique homogeneous model atmosphere which removes all anomalies and hence we became convinced to observe abundance stratification.

- Effects of pulsation on spectral lines: High temporal and spectral resolution spectroscopic time series of at least 3 roAp stars showed that Pr III and Nd III lines have the highest radial velocity pulsation amplitudes of up to $1 \mathrm{~km} \mathrm{~s}^{-1}$ (see Kochukhov \& Ryabchikova, these proceedings) which is 5 to 8 times larger than for, e.g. Ca and $\mathrm{Ba}$. An abundance stratification in the atmosphere would also explain the smaller-but nevertheless detectable- $\mathrm{RV}$ amplitudes derived from the lines of first REE ions which are formed deeper in a stratified atmosphere.

Another implication of pulsation is the need for different macroturbulence values when fitting spectral lines formed at different optical depths. In the case of $\gamma \mathrm{Equ}$, e.g., we find values ranging from 2 to $10 \mathrm{~km} \mathrm{~s}^{-1}$.

\section{Outlook}

Although the observational data collected at the SAAO, McDonald and Crimean Astrophysical Observatories is substantial, a clear boundary between roAp and noAp stars cannot yet be drawn. Only one noAp star, HD 184471 has an effective temperature which falls in the middle of the roAp star range. It obviously will be necessary to observe (and find) more non-pulsating stars in the given parameter space to answer this important question.

The spectroscopic signature found for roAp star atmospheres and which we have tried to summarize in this presentation would allow to discriminate roAp stars from non pulsating Ap stars, hence facilitating their discovery. Furthermore, we can hope to obtain additional clues to understand what drives pulsation in roAp stars.

Finally, with the evidence of different RV amplitudes due to pulsation for lines formed in different atmospheric depths we might have a powerful tool available to probe the velocity fields in three dimensions!

Acknowledgments. This work was supported by the Fonds zur Förderung der wissenschaftlichen Forschung (projects $S 7003-A S T$ and P14984) and by the Swedish Naturvetenskapliga forskningsrådet. T.R. also thanks the Russian National Program 'Astronomy' for partial financial support (grant 1.4.1.5).

\section{References}

Cowley, C.R., Ryabchikova, T., Kupka, F., Bord, D.J., Mathys, G., \& Bidelman, W.P. 2000, MNRAS, 317, 299

Cowley, C.R., Hubrig, S., Ryabchikova, T.A., Mathys, G., Piskunov, N., \& Mittermayer, P. 2001, MNRAS, 302, 381

Kupka, F., Ryabchikova, T.A., Weiss, W.W., Kuschnig, R., Rog1, J., \& Mathys, G. 1996, A\&A, 308, 886

Ryabchikova, T., Savanov, I.S., Hatzes, A.P., Weiss, W.W., \& Handler, G., 2000, A\&A, 357, 981 